\title{
Congenital stationary night blindness
}

INSERM

\section{Source}

INSERM. (1999). Orphanet: an online rare disease and orphan drug data base. Congenital stationary night blindness. ORPHA:215

\# 8805;-10.00 D)), nystagmus, strabismus, normal color vision and fundus abnormalities. 